\title{
The relationship between magnetic field strength and loop lengths in solar coronal active regions
}

\author{
R. Jain ${ }^{1}$ and C. H. Mandrini ${ }^{2, \star}$ \\ 1 Department of Applied Mathematics, University of Sheffield, S3 7RH, UK \\ e-mail: R.Jain@sheffield.ac.uk \\ 2 Instituto de Astronomía y Física del Espacio, CONICET-UBA, CC. 67 Suc. 28, 1428 Buenos Aires, Argentina \\ e-mail: mandrini@iafe.uba.ar
}

Received 13 June 2005 / Accepted 19 December 2005

\section{ABSTRACT}

By assuming that coronal active regions are made up of many loops, we investigate the relationship between the average magnetic field strength and loop length for a sample of active regions observed by the Soft X-ray Telescope aboard Yohkoh. We use photospheric magnetic data from the Michelson Doppler Imager and compute extrapolated field lines that match the observed soft X-ray emission. We compare our results with a previous study and find them to be consistent and a logical extension of such work. Such studies have important implications for solar coronal heating models.

Key words. Sun: magnetic fields - Sun: corona

\section{Introduction}

Since it was realised that the Sun's corona is hotter than the underlying photosphere by almost three orders of magnitude, there have been efforts to explain why. Dissipation of magnetohydrodynamic (MHD) waves and dissipation of stressed, current-carrying magnetic fields are among the plausible ideas. However, the major difficulty in identifying the heating mechanism is that the small scale lengths $(<1 \mathrm{~km})$ involved in coronal heating models cannot be directly verified by observations.

The solar corona consists of magnetic structures with different characteristics (active regions, coronal holes, etc.). High resolution magnetograms together with EUV and X-ray images of the corona clearly suggest a strong connection between the magnetic field and coronal heating, but the underlying processes have yet to be identified.

In recent years, there have been efforts to use observed characteristics of coronal loops in active regions (ARs) to derive various scaling laws relating different physical quantities that directly or indirectly contribute to the heating rate. Although the details of the energy release process in the corona do not depend on the loop length or the large-scale coronal magnetic field, the main aim of such studies is to compare the observed dependence of the heating rate on some fundamental physical quantities with similarly expressed dependencies derived from coronal heating theories. In this way, the number


(CONICET). of free parameters in the scaling law for the heating can be reduced.

The purpose of our study is to resolve the discrepancy between the conclusions of two such earlier analyses, by Mandrini et al. (2000) (hereafter referred to as MDK (2000)) and Yashiro \& Shibata (2001) (hereafter referred to as YS (2001)), regarding their implications for coronal heating. Both these studies found a best-fit scaling law for the heating rate of the form $H \propto L^{b}$, where $L$ is a characteristic (AR or loop) length, but show a discrepancy in the value of the exponent $b$. In the present paper we test and verify that the cause of this discrepancy is the different methods used to derive the relationship between the mean magnetic field strength $B$ and $L$ in these two studies.

MDK (2000) carried out extrapolation studies on 14 active regions (ARs) to investigate how the mean magnetic field strength of the loops depends on their end-to-end lengths. They used photospheric magnetograms, obtained with several ground-based magnetographs, to compute linear force-free and magnetostatic models and determined the average (over the tube volume) coronal field strength, $\langle B\rangle$, in approximately a thousand individual flux loops (or tubes) per AR with regularly spaced footpoints. They found a scaling law for $\langle B\rangle$ as a function of the loop lengths, $L$ (for limited ranges of lengths centered around $50-300 \mathrm{Mm})$ as: $\langle B\rangle \propto L^{\delta}$ where $\delta=-0.9 \pm 0.3$. This information, together with the findings of Porter \& Klimchuk (1995), enabled MDK (2000) to estimate the heating rate per unit volume as a function of $\langle B\rangle$ using 
a quasi-static model for coronal loops. MDK (2000) also compared these rates derived from observations with the heating rates predicted by various theoretical models, considering cases with and without coronal and photospheric quantities being identical. They concluded that models based on the dissipation of stressed, current-carrying magnetic fields are in better agreement with the observations than models that attribute coronal heating to the dissipation of MHD waves injected at the base of the corona. A similar conclusion was reached by the independent study of Démoulin et al. (2003) who used the results derived from the long-term evolution study of an isolated AR (AR 7978) by van Driel-Gesztelyi et al. (2003). The two studies, Démoulin et al. (2003) and van Driel-Gesztelyi et al. (2003), combined Soft X-ray Telescope (SXT, Tsuneta et al. 1991) observations of the full AR and the more precise plasma diagnostic given by the Bragg Crystal Spectrometer (BCS, Culhane et al. 1991) aboard Yohkoh, with magnetic field measurements obtained with the Michelson Doppler Imager (MDI, Scherrer et al. 1995) aboard the Solar and Heliospheric Observatory (SoHO). They were able to analyse a larger sample of magnetic field strengths and plasma parameters in different conditions with less statistical noise.

YS (2001) also studied the relationship between thermal and magnetic properties of 31 mature active regions observed with SXT. They integrated the soft X-ray emission over the entire AR and obtained a single temperature for each AR using the filter ratio technique. YS (2001) (see also Jain \& Yashiro 2002) found that for their sample of ARs the mean magnetic flux density is independent of the region size. This led YS (2001) to conclude that their results were consistent with the Alfvén wave heating mechanism and possibly the nanoflare model proposed by Sturrock et al. (1999) for strong magnetic fields.

The discrepancy between the results of MDK (2000), $B$ $\frac{1}{L}$, and YS (2001), $B$ independent of $L$, needs to be explained since it is unlikely that a different main heating mechanism is at work in ARs with similar global characteristics. The explanation could lie in the different methods used to estimate the typical scale length and the magnetic flux density to derive their relationship. Unlike MDK (2000), YS (2001) did not carry out extrapolation studies. The typical length scale in YS (2001) study is the AR size, which is taken as the square root of its area $(A)$, and ranges between $[10,100] \mathrm{Mm}$. Also, the mean magnetic flux density for each coronal active region was calculated by assuming that the total photospheric magnetic flux $(\phi)$, measured from MDI/SoHO magnetograms at the photospheric level is the same as at the coronal level. YS (2001) thus used the coronal active region area, $A$, to obtain the mean magnetic flux density as $B=\phi / A$ for each AR.

A specific aim of this paper is to carry out extrapolation studies for a subset of the ARs considered by YS (2001), in the same way as MDK (2000) did, and determine the relationship, if any, between loop length and average field strength of the loop. The paper is organised as follows. In Sect. 2, we describe the data analysis and our results. In Sect. 3, we present our conclusions.

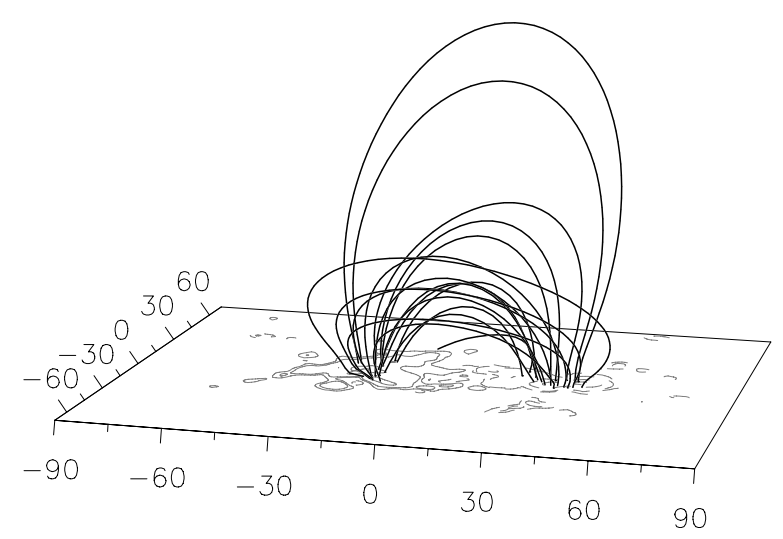

Fig. 1. Side view of the extrapolated field lines showing their height for AR7968.

\section{Data analysis and results}

All the coronal data used in this study are from full frame SXT images obtained either with the thin A11 or A1Mg filters. For the magnetic data we use the full disc level 1.5 MDI magnetograms. These data are the average of 5 magnetograms with a cadence of $30 \mathrm{~s}$. They are constructed once every $96 \mathrm{~min}$. The error in the flux densities per pixel in the averaged magnetograms is $\approx 9 \mathrm{G}$, and each pixel has a mean area of $1.96 \mathrm{Mm}^{2}$.

The field lines are computed under the linear force-free assumption:

$\nabla \times \boldsymbol{B}=\alpha \boldsymbol{B}$

(with $\alpha$ constant, in particular $\alpha=0$ for the potential case) using a fast Fourier transform method (see Alissandrakis 1981; Démoulin et al. 1997). In brief (see Green et al. 2002, for details), the value of $\alpha$ is determined by an iterative process. As a first step, the field lines are computed for a given $\alpha$. We then calculate the mean distance between the observed SXT coronal loop or the intensities on a series of points (when loops are not clearly visible in SXT images). The process is repeated with iteration on $\alpha$ until the lowest mean distance (the best global fit) is achieved. Figure 1 shows a side view of the computed magnetic field lines for active region AR 7968. The extrapolated field lines globally match the soft X-ray emission observed by SXT (see Fig. 2).

In Table 1 we list the ARs (in order of magnetic complexity) used in this study and the important parameters obtained from observations ( $\Phi$ and $S_{\mathrm{AR}}$ ) and magnetic field modeling $(\alpha)$. In this study, $\Phi$ is the average between the positive and the absolute value of the negative magnetic flux at the photospheric level. The quantity $S_{\mathrm{AR}}$ is the active region size which, in our case, is the flux weighted mean distance between opposite polarity photospheric fields given as follows (see MDK 2000):

$S_{\mathrm{AR}}^{2}=\left(X_{\mathrm{p}}-X_{\mathrm{n}}\right)^{2}+\left(Y_{\mathrm{p}}-Y_{\mathrm{n}}\right)^{2}$

$X_{\mathrm{p}}=\frac{\sum x B_{z}(>0)}{\sum B_{z}(>0)}, \quad Y_{\mathrm{p}}=\frac{\sum y B_{z}(>0)}{\sum B_{z}(>0)}$.

Similar expressions for $X_{\mathrm{n}}$ and $Y_{\mathrm{n}}$ are given for the mean position of the negative concentrations $\left(B_{z}<0\right)$. In both cases only 

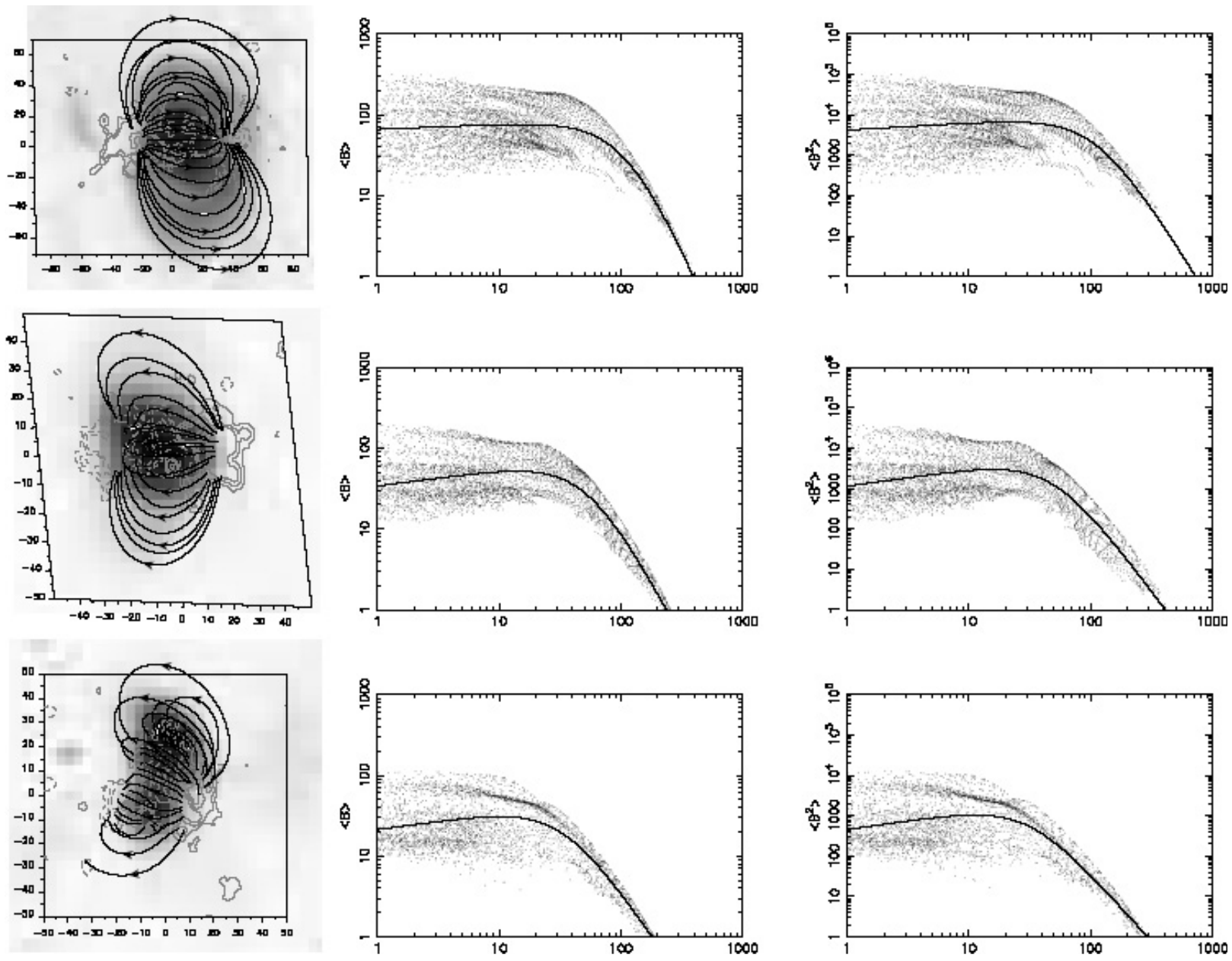

Fig. 2. First column: an overlay of an SXT image on an MDI magnetogram including computed field lines for three of the modelled ARs. From top to bottom: AR 7968 (magnetic data at 14:28 UT and SXT AlMg filter image at 18:08 UT on June 7, 1996), AR 7994 (magnetic data at 06:27 UT and SXT AlMg filter image at 06:23 UT on November 11,1996) and AR 8024 (magnetic data at 19:13 UT and SXT AlMg filter image at 19:09 UT on March 20, 1997). Three $( \pm 20,50,100 \mathrm{G})$ isocontours of the line of sight magnetic field have been drawn for AR 7994 and AR 8024 and four $( \pm 40,100,500,1000 \mathrm{G})$ for AR 7968. The $x$ and $y$ axes are in Mm. Second and third columns: scatter plots of $\langle B\rangle$ and $\left\langle B^{2}\right\rangle$ for the three chosen ARs. The axes have logarithmic scales with the field line length $L$ (abscissa) measured in Mm and the magnetic field in G. Each point represents a computed field line. Figures for $\langle B\rangle$ and $\left\langle B^{2}\right\rangle$ correspond to field lines anchored at places where the absolute value of the field at both footpoints is larger than $10 \mathrm{G}$. The curve in each plot is a least-square fit to the function $F_{1}(L)$ to the points (see Eq. (4)).

vertical field strengths $\left.\left|B_{z}\right|\right\rangle 10 \mathrm{G}$ are included to avoid noisy data.

From the modelled magnetic fields for every AR, we compute the flux-tube volume averaged magnetic quantities $\langle B\rangle$ and $\left\langle B^{2}\right\rangle$ as (see MDK 2000):

$$
\begin{aligned}
\langle B\rangle=\frac{\int B \mathrm{~d} V}{\int \mathrm{d} V} \approx \frac{L}{\int \mathrm{d} s / B}, \\
\left\langle B^{2}\right\rangle=\frac{\int B^{2} \mathrm{~d} V}{\int \mathrm{d} V} \approx \frac{\int B \mathrm{~d} s}{\int \mathrm{d} s / B},
\end{aligned}
$$

where $\mathrm{d} V$ is the elemental volume, $s$ is the curvilinear coordinate along the central axis of the tube, and $L=\int \mathrm{d} s$ is the flux-tube length. Under the assumption that the variation of $B$ within the cross section of the tube is negligible, we can replace the volume integral by a line integral using the magnetic flux conservation.
In Fig. 2, first column, we show the extrapolated field lines superimposed on the X-ray images of three ARs. The ARs, in the particular set chosen by YS (2001), are in general decaying ARs in which individual loops can barely be distinguished, and the soft X-ray images come from SXT full disc data, which have a low spatial resolution $(\sim 5 \mathrm{arcsec})$. Thus, our fit is not done to isolated loops but to the global SXT emission. Different aspects of two of the ARs included in Table 1 (AR 7968 and AR 7981) have been studied by Deng et al. (1999), Mandrini et al. (2001), Démoulin et al. (2003) and Mandrini et al. (2004). The values of $\alpha$ mentioned in Table 1 for these two ARs are the same as in these studies. In general, when loops cannot be distinguished, $\alpha$ can vary by about $20 \%$ but the computed field lines still follow the global trend of the X-ray emission; for larger variations in $\alpha$, the discrepancies become visible.

In the second and third columns of Fig. 2, we show $\langle B\rangle$ and $\left\langle B^{2}\right\rangle$ as a function of $L$ (in logarithmic scale and measured in $\mathrm{Mm}$ ), respectively. In order to quantify the functional 

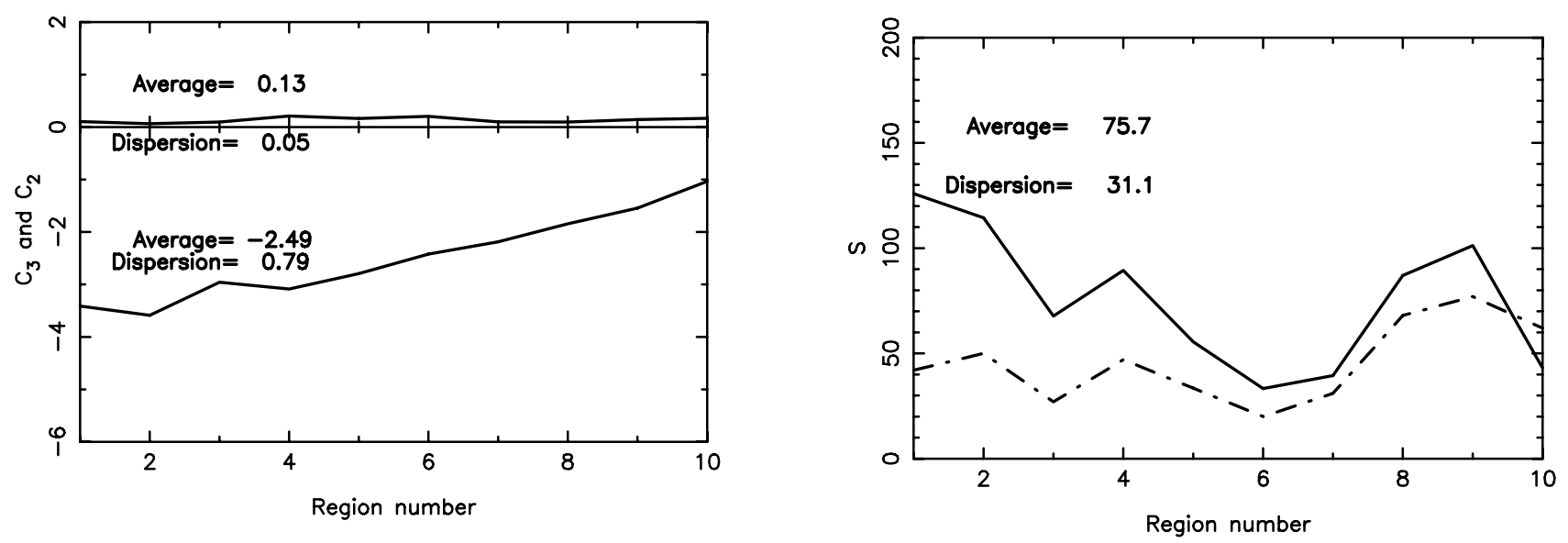

Fig. 3. Results from fitting function $F_{1}$ to $\langle B\rangle$ scatter plot data for the 10 ARs selected from YS (2001). Coefficents $C_{2}$ and $C_{3}$ are plotted on the left and parameter $S$ on the right panels, respectively. The dash-dotted line in the right panel represents the estimated active region size $S_{\mathrm{AR}}$ as defined in the text (see Eqs. (2)).

Table 1. SXT active regions and relevant parameters.

\begin{tabular}{ccccc}
\hline \hline $\begin{array}{c}\text { Region } \\
\text { number }\end{array}$ & $\begin{array}{c}\text { NOAA } \\
\text { number }\end{array}$ & $\begin{array}{c}\Phi^{a} \\
\left(10^{21} \mathrm{Mx}\right)\end{array}$ & $\begin{array}{c}S_{\mathrm{AR}}^{b} \\
(\mathrm{Mm})\end{array}$ & $\begin{array}{c}\alpha^{c} \\
\left(10^{-3} \mathrm{Mm}^{-1}\right)\end{array}$ \\
\hline 1 & 8052 & 3.2 & 42 & 9.4 \\
2 & 7968 & 4.0 & 50 & -6.3 \\
3 & 7961 & 1.3 & 27 & 0 \\
4 & 7982 & 4.5 & 38 & 0 \\
5 & 7994 & 0.9 & 33 & 0 \\
6 & 8024 & 0.7 & 20 & 0 \\
7 & 8041 & 0.5 & 31 & 0 \\
8 & 7981 & 12.3 & 68 & 6.3 \\
9 & 7999 & 15.9 & 77 & -12.3 \\
10 & 8004 & 9.0 & 62 & -12.3 \\
\hline
\end{tabular}

${ }^{a} \Phi$ is the unsigned magnetic flux at the photospheric level (see the text).

${ }^{b} S_{\mathrm{AR}}$ is the active region size which is the flux weighted mean distance between opposite polarity photospheric fields (see the text).

${ }^{c} \alpha$ is the shear parameter as shown in Eq. (1).

dependence of $\log \langle B\rangle$ and $\log \left\langle B^{2}\right\rangle$ on $L$, we follow MDK (2000) and propose the following expression:

$F_{1}(L)=C_{1}+C_{2} \log L+\frac{C_{3}}{2} \log \left(L^{2}+S^{2}\right)$

where $F_{1}$ represents either $\log \langle B\rangle$ or $\log \left\langle B^{2}\right\rangle$. The four parameters $C_{1}, C_{2}, C_{3}$ and $S$ are unique for each AR. In particular, $S$ is related to some typical AR length, as discussed below (see also MDK (2000) Sects. 3.1 and 3.2). When $F_{1}=\log \langle B\rangle$, Eq. (4) simplifies to the power law $\langle B\rangle \propto L^{\delta}$ in the two limits $L \ll S$ and $L \gg S$, where $\delta=C_{2}$ and $\delta=C_{2}+C_{3}$, respectively. The least-squares fit $F_{1}(L)$ is plotted as a solid line in the second and third column of Fig. 2 for $\langle B\rangle$ and $\left\langle B^{2}\right\rangle$, respectively. We have chosen the loop lengths in the interval $[1,1000] \mathrm{Mm}$, and the photospheric field strength to be between $[10,5000]$ G. Figure 2 shows that for small values of $L$, there are more data points and a larger scatter suggesting that $\langle B\rangle$ and $\left\langle B^{2}\right\rangle$ are almost independent of $L$. Very low values of $L$ do not represent real SXT coronal loops since they link mixed photospheric polarities which are at the noise level. However, we have decided to keep the full range of lengths and magnetic field strengths to illustrate the general behaviour and also to simplify the comparison with MDK (2000) results. On the other hand, there are fewer loops of longer lengths, and $\langle B\rangle$ and $\left\langle B^{2}\right\rangle$ show a clear decrease with $L$. The qualitative behaviour seen in Fig. 2 is present in all the ARs listed in Table 1. Some differences in the individual distributions are related to the magnetic characteristics of each AR, e.g. number, intensity and relative position of the flux concentrations at the photosphere.

Figure 3 shows the parameters $C_{2}, C_{3}$ and $S$, obtained from fits to the $\langle B\rangle$ versus $L$ scatter plot data for the different ARs as a function of region number from Table 1 . The averages and dispersions shown in each panel of this figure indicate the mean values and standard deviations of each represented parameter. We see from Fig. 3 (left panel) that $C_{2}$ hovers very close to 0 in all cases, indicating that the average field strength is nearly independent of length for $L \leq S . C_{3}$ ranges mostly between -3.5 and -1 and tends to be less negative for more complex regions. All these results are compatible with the results found by MDK (2000).

The parameter $S$ (Fig. 3, right panel) varies from about 40 to $130 \mathrm{Mm}$ and is related to a typical AR length. In order to show the relationship between $S$ and $S_{\mathrm{AR}}$, we have drawn them together with solid and dash-dotted lines, respectively. It can be seen that there is a reasonable correlation between both parameters. In general, $S$ is about twice $S_{\mathrm{AR}}$ in simple bipolar configurations but becomes close to it as the complexity of the region increases. This difference can be explained by noticing that $S_{\mathrm{AR}}$ only characterizes the dipolar aspects of the photospheric flux distribution. When a magnetic configuration is complex and has intermixed polarities, $S_{\mathrm{AR}}$ does not necessarily reflect their separation. For example, in the extreme case of a region of fully mixed field, as in the quiet Sun during solar minimum, the value of $S_{\mathrm{AR}}$ will be zero, even though there is a characteristic separation between opposite polarity elements. The relationship between $S$ and $S_{\mathrm{AR}}$ shown in Fig. 3 is very 

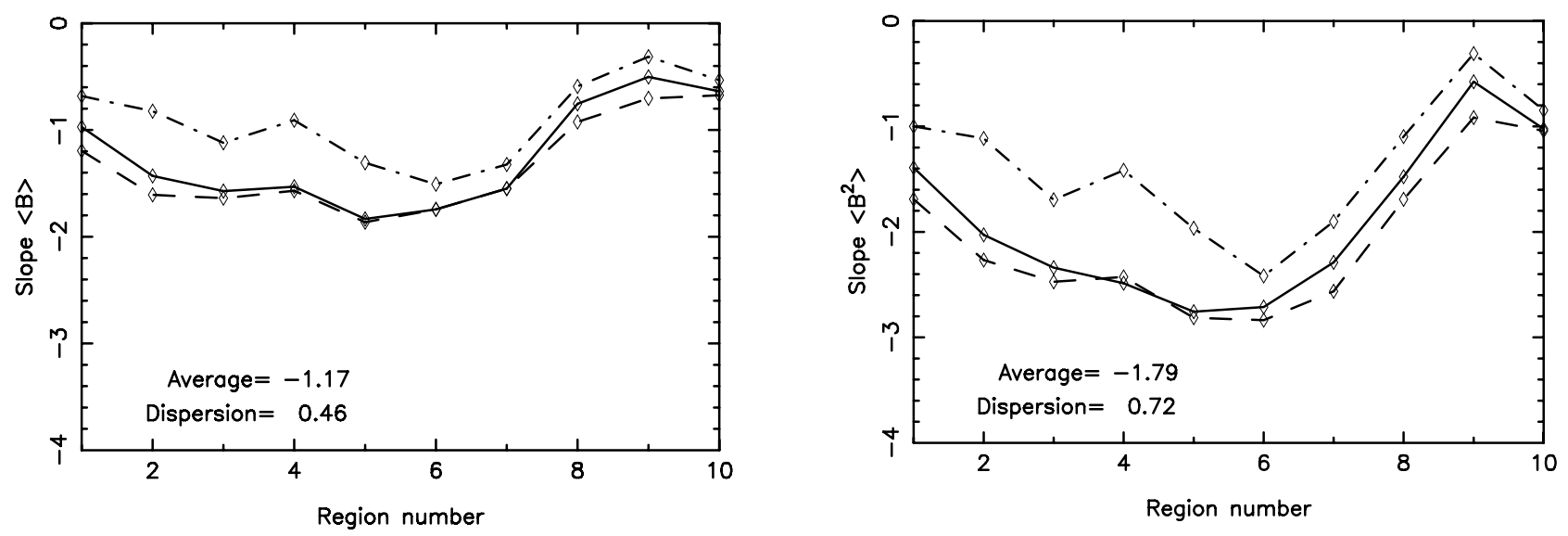

Fig. 4. Results from fitting the linear function $F_{2}$ to the scatter plot data for the 10 ARs in Table 1 . The slope $\delta$ is plotted against the active region number for $\langle B\rangle$ (left panel) and $\left\langle B^{2}\right\rangle$ (right panel). The solid curve is for the range of lengths [40,200] Mm, while the dash-dotted and dashed curves are for the ranges $[20,200]$ and $[40,300]$, respectively.

Table 2. Average values of $C_{3}$ and correlation factors $(r)$ to $F_{1}$.

\begin{tabular}{lcc}
\hline \hline Averages & $\langle B\rangle$ & $\left\langle B^{2}\right\rangle$ \\
\hline$C_{3}$ & $-2.49 \pm 0.79$ & $-3.76 \pm 0.93$ \\
$r$ & $0.62 \pm 0.14$ & $0.53 \pm 0.14$ \\
\hline
\end{tabular}

similar to the one found between the same parameters by MDK (2000) for their particular set of ARs. We note that the range of $S$ is smaller for our set than for the MDK (2000) set, indicating that YS (2001) ARs are much less extended. This fact will be reflected in the range of loop lengths representing the soft X-ray emission (see below).

We have analysed similar plots for $\left\langle B^{2}\right\rangle$ and find that the results are similar: a very flat distribution for $L \ll S\left(C_{2} \approx 0\right.$.) and a steeply declining section for large values of $L$. The main difference is that the coefficient $C_{3}$ is more negative for $\left\langle B^{2}\right\rangle$. The average values and the dispersions of $C_{3}$ for the 10 ARs are shown in the first row of Table 2, for the scatter plots of $\langle B\rangle$ and $\left\langle B^{2}\right\rangle$. The quality of the least-squares fits to $F_{1}$ (with $F_{1}=\log \langle B\rangle$ or $\left.F_{1}=\log \left\langle B^{2}\right\rangle\right)$, indicated by the Pearson's correlation factor $(r)$, is also given (averaged over 10 ARs).

The general law discussed above involves parameters that vary from one AR to the next (primarily $S$ and $C_{3}$ ). However, MDK (2000) found a law linking $L$ and $\langle B\rangle$ and $\left\langle B^{2}\right\rangle$ based on parameters that were the same for all the cases they studied. From the scatter plots for $F_{1}(L)$, we have seen that this function reduces to a linear shape in the limits $L \ll S$ and $L \gg S$. Therefore, following MDK (2000), we test if a power law can be applied over a limited range of intermediate lengths $(L \approx S)$ and consider the simplest case, $B \propto L^{\delta}$. We fit the individual AR (taking limited intervals for the loop lengths) distributions to the function:

$F_{2}(L)=C+\delta \log L$

where for $F_{2}=\log \langle B\rangle,\langle B\rangle=10^{C} L^{\delta}$ (similarly for $\log \left\langle B^{2}\right\rangle$ ).

The range of lengths for which $F_{2}$ was tested by MDK (2000) was the range of SXT loop lengths used in the Porter \& Klimchuk (1995) study. In this latter paper the lengths
Table 3. Average values of the slopes and correlation factors $(r)$ to $F_{2}$.

\begin{tabular}{lcc}
\hline \hline Averages & $\langle B\rangle$ & $\left\langle B^{2}\right\rangle$ \\
\hline$\delta$ & $-1.17 \pm 0.46$ & $-1.79 \pm 0.72$ \\
$r$ & $0.69 \pm 0.19$ & $0.61 \pm 0.21$ \\
\hline
\end{tabular}

of SXT loops were measured individually and were in the $[50,300] \mathrm{Mm}$ range, which was the central range considered by MDK (2000). However, for the set of ARs in our study, we are unable to identify individual loops in most of the ARs. Our ARs are spatially less extended than those of MDK (2000) suggesting that the soft X-ray emission is, in general, restricted to shorter field lines and, hence, the loop length range $[50,300] \mathrm{Mm}$ is rather large. Therefore, if we compare our range of $S([40,130] \mathrm{Mm})$ to that of MDK (2000) $([40,240] \mathrm{Mm})$, it is evident that we should modify our range of lengths to determine the value of $\delta$. The same is true if we compare the average value of $S_{\mathrm{AR}}$ for our set $(46 \mathrm{Mm})$ with the average $S_{\mathrm{AR}}$ for MDK (2000) (60 Mm). To choose the range of $L$ we proceed as follows. We rescale the central range of MDK (2000), i.e. [50,300] Mm, by multiplying by a factor of our average $S_{\mathrm{AR}}$ divided by the average $S_{\mathrm{AR}}$ of MDK (2000). This gives our central range to be approximately [40,200] Mm. Next, we consider SXT emission over $10 \%$ of the maximum intensity of each $\mathrm{AR}$ and make sure that in each AR there is a significant number of field lines within [40,200] Mm. Because our ARs have different extensions, we consider two intervals around this central one, [40,300] Mm and [20,200] Mm. Figure 4 (left and right panels) shows $\delta$ as a function of AR number for these three intervals of $L$ ([20,200], [40,200], $[40,300] \mathrm{Mm})$ for $\langle B\rangle$ and $\left\langle B^{2}\right\rangle$, respectively. For all three intervals, we consider the absolute value of the photospheric field strength at both loop feet to be above $10 \mathrm{G}$. The averages and dispersions in Fig. 4 correspond to the average $\delta$ for all the three intervals, i.e. the average of all thirty $\delta$ values included in the figure and its dispersion.

The value found for $\delta$ for our particular set of ARs in the case of $\langle B\rangle, \delta=-1.2 \pm 0.5$, is in good agreement within error 
bars with the value reported by MDK (2000), i.e. $-0.9 \pm 0.3$ (see Sect. 5.5 in MDK (2000)). The difference is believed to be due to different magnetic complexities of the ARs in our subset, when compared to those of MDK (2000). Most of our ARs are weak field bipolar regions, only 3 are clearly quadrupolar. In MDK (2000) most of the ARs are quadrupolar and, generally, have a much stronger field. The two studies now firmly suggest that a law for the scaling of $\langle B\rangle$ with $L$ exists in solar coronal ARs; the value of the parameter $\delta$ in this law depends on the degree of complexity of the ARs included in the set under analysis.

\section{Discussion and conclusions}

We find that there exists a power law relation between $\langle B\rangle$ and $\left\langle B^{2}\right\rangle$ and $L$ for solar coronal AR loops, contrary to what was shown by YS (2001), who found that the mean magnetic field intensity and loop lengths were not related. We conclude that the discrepancy between our result and those of YS (2001) is due to the different methods used in estimating the parameters in the power law in the respective studies. In our study (see also MDK 2000), we estimate the average coronal field magnitudes using a volume integration along flux tubes computed from an extrapolated coronal field (mainly Eqs. (3)); the lengths used to estimate the scaling law correspond to the range of lengths of the computed field lines for which the SXT emission is over a certain threshold (see Sect. 2). In this way the characteristics (configuration and complexity) of each AR are better taken into account. In YS (2001) the typical length scale was the AR size, and is taken as the square root of its area $(A)$; the mean magnetic coronal flux density was calculated by taking the total photospheric magnetic flux (measured by SoHO/MDI) divided by the AR area. For future studies, similar to the study presented here, it is our conclusion that estimating mean quantities in a global way seldom reflects the actual complexities of the magnetic configuration in the corona and, when possible, should be avoided.

The power law of the form $L^{\delta}$ provides a good statistical fit for both $\langle B\rangle$ and $\left\langle B^{2}\right\rangle$ in a limited range of lengths. This was also found by MDK (2000). The range of $L$ obviously depends on the size of the active regions. Because of the smaller active regions in our study compared to those studied by MDK (2000), we have scaled down the range of lengths that we take to derive the power law index $\delta$. However, even with a different range of lengths in our set, the value of $\delta$ does not show very significant changes; the difference between the $\delta$ value here and in MDK (2000) is mainly due to the number of complex ARs included in each set.

We can now combine the 10 ARs of our study with the 14 ARs of MDK (2000) to have a set that includes ARs of varying sizes and complexities, and find a single value for $\delta$. We recomputed $\delta$ for the same three loop length ranges that were considered by MDK $(2000)([30,300] \mathrm{Mm},[50,300] \mathrm{Mm}$ and $[50,400] \mathrm{Mm})$, noticing that the dispersion of $\delta$ increases for our 10 ARs. We found that $\delta=-1.07 \pm 0.43$ for $\langle B\rangle$ and $-1.64 \pm 0.70$ for $\left\langle B^{2}\right\rangle$ (these values are the averages and dispersions of the 72 values of $\delta$, i.e for each of the 24 ARs considering three ranges of loop lengths). If we use any of these $\delta$ values to derive the scaling law for the heating rate as a function of $L$ for different coronal heating models, we find similar scalings, within error bars, as those found by MDK (2000) and Démoulin et al. (2003). Furthermore, if these scalings derived from models are then compared with the observed heating rate obtained from observations using a quasi-static model for coronal loops, as was done by MDK (2000) and Démoulin et al. (2003) (see also Mandrini 2005, for a review), we find that models based on the dissipation of stressed, current-carrying magnetic fields are in better agreement with SXT observations than the models that involve dissipation of MHD waves, even for a set of ARs having most diverse characteristics.

Findings of studies like the one described here when combined with quasi-static models of coronal loops, to derive heating-rate scalings for coronal heating models, should be used with care. Observations of cooler loops from the Extreme Ultraviolet Imaging Telescope (EIT/SoHO) and the Transition Region and Coronal Explorer (TRACE) suggest that many loops with temperatures around $1 \mathrm{MK}$ are not in static equilibrium. Some are seen to evolve rapidly and even those that appear steady tend to have densities much higher than can be explained by equilibrium theory (Aschwanden et al. 2000). Aschwanden et al. (2001) found that about one-third of the TRACE loops in their study can be explained by equilibrium models if the heating is sufficiently concentrated near the foot points, but two-thirds cannot be. Winebarger et al. (2003) suggest that the fraction compatible with equilibrium may actually be smaller. SXT observe loops that are generally much hotter than $1 \mathrm{MK}$. In addition to being hotter, they are also broader and fuzzier in appearance, which is not an artifact of the instrumental resolution. Furthermore, TRACE and EIT loops are overdense relative to equilibrium, SXT loops either have the correct density or are underdense. As described in Porter \& Klimchuk (1995), most SXT loops are compatible with equilibrium for reasonable values of the filling factor. This indicates that, while there can be no guarantee that SXT loops are in quasi-static equilibrium, the observations are consistent with such an interpretation. The presence or absence of other heating mechanisms will be identified with better spatial and spectral resolution and coverage with future missions such as Solar B.

Acknowledgements. We thank Dr. S. Yashiro for providing us with his active region list and SXT observations. RJ acknowledges The Nuffield Foundation for NUF-NAL 04 award. We are also grateful to The Royal Society for their financial support. We thank the SoHO/MDI consortium for the magnetic field data and the Yohkoh team for SXT data. SoHO is a joint project by ESA and NASA. C.H.M. thanks the Argentinean grants: UBACyT X329, PICT 12187 (ANPCyT) and PIP 6220 (CONICET).

\section{References}

Alissandrakis, C. E. 1981, A\&A, 100, 197

Aschwanden, M. J. 2000, ApJ, 531, 1129

Aschwanden, M. J., Schriver, C. J., \& Alexander, D. 2001, ApJ, 550, 1036

Culhane, J. L., Bentley, R. D., Hiei, E., et al. 1991, Sol. Phys., 136, 89

Démoulin, P., Bagalá, L. G., Mandrini, C. H., Hénoux, J. C., \& Rovira, M. G. 1997, A\&A, 325, 305 
Démoulin, P., Mandrini, C. H., van Driel-Gesztelyi, L., et al. 2002, A\&A, 382, 255

Démoulin, P., van Driel-Gesztelyi, L., Mandrini, C. H., Klimchuk, J. A., \& Harra, L. 2003, ApJ, 586, 592

Deng, Y. Y., Schmieder, B., Mandrini, C. H., et al. 1999, A\&A, 349, 927

Green, L. M., López Fuentes, M., Mandrini, C. H., et al. 2002, Sol. Phys., 208, 43

Jain, R., \& Yashiro, S. 2002, A\&A, 394, 1111

Mandrini, C. H. 2005, AIP Conf. Proc., 784, 103

Mandrini, C. H., Démoulin, P., \& Klimchuk, J. A. 2000, ApJ, 530, 999

Mandrini, C. H., Démoulin, P., Schmieder, B., Deng, Y. Y., \& Rudawy, P. 2002, A\&A, 391, 317
Mandrini, C. H., Démoulin, P., van Driel-Gesztelyi, L., et al. 2004, Ap\&SS, 290, 319

Porter, L. J., \& Klimchuk, J. A. 1995, ApJ, 454, 499

Scherrer, P. H., Bogart, R. S., Bush, R. I., et al. 1995, Sol. Phys., 162, 129

Sturrock, P. A. 1999, ApJ, 521, 451

Tsuneta, S., Acton, L., Bruner, M., et al. 1991, Sol. Phys., 136, 37

van Driel-Gesztelyi, L., Démoulin, P., Mandrini, C. H., Harra, L., \& Klimchuk, J. A. 2003, ApJ, 586, 579

Winebarger, A. R., Warren, H. P., \& Mariska, J. T. 2003, ApJ, 587, 439

Yashiro, S., \& Shibata, K. 2001, A\&A, 550, L113 